ANNUAL REPORT , 1999

Project Title: MINIATURE NUCLEAR MAGNETIC RESONANCE SPECTROMETER FOR IN-SITU AND IN-PROCESS ANALYSIS AND MONITORING, Grant\# 97ER62514

Publication Date: $\quad$ September 15, 1999

\section{Lead Principle Investigator:}

Gennady Friedman, Associate Professor, The University of Illinois at Chicago

Department of Electrical Engineering

and Computer Science, Mail Code 154

851 South Morgan Street

Chicago, IL 60607

\section{Co-Investigator}

Alan Feinerman, Associate Professor, The University of Illinois at Chicago

Department of Electrical Engineering and Computer Science, Mail Code 154

851 South Morgan Street

Chicago, IL 60607

Graduate Students: Eric Growney (MS), Lisa Murphy (MS), Muqu Sun (PhD),Samuel Grant (PhD),Yikun Huang $(\mathrm{PhD})$

\title{
RESEARCH OBJECTIVES.
}

The main purpose of this research is to develop a nuclear magnetic resonance (NMR) spectrometer that is small enough to be held in the palm of a hand. Such an instrument could be used in many applications of importance to DOE as well as to industry. These applications would include in-field characterization of contaminants, monitoring and analysis of chemical processes in chemical plants and other environmental monitoring applications. In addition to having drastically reduced size and cost, a miniature NMR instrument would be ideally suited for chemical analysis of volume limited samples which frequently occur in biological, medical and drug development applications.

\section{RESEARCH PROGRESS AND IMPLICATIONS}

As of June $15^{\text {th }}$, 1998 several important results have been obtained. First of all, the miniature NMR probe which was built in the first year of the project was analyzed and tested for various properties. Secondly, design of the miniature permanent magnet for NMR started in the first year of the project was finalized and its fabrication was initiated. Lastly, preliminary designs of the shimming coils were evaluated.

To appreciate the significance of the various NMR components mentioned above to the entire NMR spectrometer it is worth briefly reviewing the basic principles of a NMR spectrometer. In NMR, nuclei of a sample that posses magnetic moments are excited into precessional motion around the direction of a static magnetic field. The frequency of this precession is proportional to the strength of the static field and is usually in the range of radio frequencies $(40-500 \mathrm{MHz})$. This precession is detected using a NMR probe, which is usually a radio frequency coil. The signal thus obtained by the NMR probe from the precession of magnetic nuclei contains frequencies not only related to the static magnetic field, but also to local molecular scale fields. This allows characterization of the collection of nuclei chemically and makes NMR spectrometer an important tool in analytical chemistry.

There are two main difficulties in most NMR spectrometers. One is related to the weakness of the NMR signal. This is usually dealt with by increasing the strength of the static magnetic field and by designing more sensitive NMR probes. The other difficulty is related to the uniformity of the static magnetic field over the extent of the sample. Non-uniform static field limits the spectral resolution and of the spectrometer and its usefulness as an analytical tool by obscuring the presence of the local molecular interactions. Field correction constant current coils called shimming coils are most frequently used to improve field uniformity.

In the miniature NMR spectrometer being developed in this project, the static magnetic field of strength 1 and 2 Tesla will be produced by a permanent magnet over the extent of about 100 cubic millimeters. The radio frequency coil acting as the NMR probe will consist of flat ribbon of copper conductor wound in a scroll around a small $(0.5 \mathrm{~mm})$ diameter capillary used to hold the liquid sample. The NMR probe and the capillary will be placed between to silicon wafers on which shimming coils will be patterned. 
Having developed and constructed 0.5 and $0.8 \mathrm{~mm}$ scroll-style NMR probes in the first year of the project, the sensitivity and spectral resolution of these probes were studied theoretically and tested experimentally this year. A semi-analytical technique was developed to analyze spectral resolution of the miniature scroll probes. This technique is based on calculation of equivalent magnetic charges induced on the surfaces of the scroll probe by the external static uniform magnetic field used to produce nuclear magnetic precession. Physically the equivalent magnetic charges are related susceptibility differences between the sample and the NMR probe. The presence of such susceptibility difference turns out to be a critical factor limiting spectral resolution in miniature NMR because the surface of the NMR probe is in much closer proximity to every point within the volume of the sample than in large scale instruments. The method of calculating non-uniformity in the static magnetic field caused by the susceptibility mismatch and of the resulting spectral distortions caused by the NMR probe appeared in [1].

In addition to developing theoretical methods that could be used to analyze spectral distortions caused by miniature NMR probes, these theoretical methods were tested experimentally this year. The experimental testing of the spectral distortions was carried out using NMR facility at the Research Resources Center at the University of Illinois at Chicago. The results of these experimental tests as well as theory and design of scroll-style NMR probes were submitted as a paper to the Journal of Magnetic Resonance. They were also part of a Master's Thesis of my student Eric Growney.

In addition to studying spectral distortions caused by miniature NMR probes, the study of sensitivity of miniature scroll and wire-style NMR probes was carried out. Analytical calculations of the signal-to-noise ratio in these probes were performed. Subsequently, Finite Element method was used to refine the calculations of the signal-to-noise ratio. These calculations required computation of radio frequency field and losses within the NMR probes. The above theoretical developments demonstrated how the NMR probes behave with varying number of layers. We are the first group to study the use of multi-layer radio frequency coils for use as NMR probes. The Finite Element calculations were compared with electrical tests of losses in scroll-style miniature NMR probes that were conducted in the first year and good agreement between theoretical predictions and the tests was found. Moreover, tests of relative signal-tonoise ratio of the NMR probes with different number of layers were performed this year using NMR data directly this year. These tests also showed very good agreement with the theoretical calculations. The results of theoretical signal-to-noise ratio calculations and comparison with NMR and electrical measurements were submitted in a paper to the IEEE Transactions on Magnetics. This work also appears partly in the Masters Thesis of my student Lisa Murphy and is also a part of $\mathrm{PhD}$ work by Samuel Grant.

In studying the problem of noise and losses in NMR probes, a general method for calculation of excess losses (losses due to high frequency currents) in conductors of arbitrary shape was developed. The main difference of this method over other methods previously employed to solve this type of problem is that it allows an easily computable estimates of losses and, therefore, of noise. This method of calculation of losses was discussed in a paper submitted to the Journal of Applied Physics and will be presented at the MMM-99 conference.

In addition to finalizing the design, analysis and experimental testing of miniature NMR probes this year work was performed on modeling of permanent magnet materials that could be used in producing static magnetic fields for NMR. One of the main difficulties in designing high uniformity NMR permanent magnets is understanding and control of permanent magnetization. That, in turn, relies on understanding of hysteresis. Work was started in the first year on the development of hysteresis model that could be used for this purpose. This work was completed this year and a paper about this work appeared [2]. The most important result of this work was that conditions were established that allows one to determine with certainty if the suggested hysteresis model is applicable to a given magnetic material. Work was also performed year on the measurement methods that could be used to identify parameters of the vector-type hysteresis model developed. This work used Vibrating Sample Magnetometer available in the laboratory of Prof. James Kouvel at the Physics Department at the University of Illinois at Chicago. The paper containing the proposed hysteresis model and measurement of its parameters was submitted to the Journal of Applied Physics. The results of this paper will also be presented at the MMM-99 conference. This work was performed with the help of my student Yikun Huang. 
Analysis of the permanent magnet structure was also finalized this year. This permanent magnet design differs from the one originally proposed (described in the original proposal). Its main advantage is that it is azimuthally symmetric and allows complete access to the NMR sample and probe from all sides as well as axially. It also allows axial spinning of the magnet to improve the apparent uniformity of the static magnetic field produced. A paper summarizing the detail of the permanent magnet design was submitted to the IEEE Transactions on Magnetics. This magnet is currently being manufactured with the assistance of the Dexter Magnetics Corporation.

Lastly, this year we began designing shimming coils that could be used for correction of static magnetic field non-uniformity. So far, it looks like planar coil array called Anderson coils will be the best for our purposes. The design and modeling of shimming coils is being carried out with the help of my student Muqu Sun.

\section{PLANNED ACTIVITIES}

We plan to finish the fabrication of the permanent magnet by February 2000. Testing of the magnet and of the magnetic field uniformity produced by this magnet will be conducted until about June 2000 . At the same time we plan to finish design and fabrication of the shimming coils by June 2000. Starting around June 2000 and until January 2001 the entire miniature NMR spectrometer will be tested.

\section{PUBLICATIONS, PRESENTATIONS AND INFORMATION ACCESS}

\section{$\underline{\text { REFEREED PUBLICATIONS }}$}

[1] E. Growney, G. Friedman, R. Gerald, "Computation of distortions in magnetic field and spectrum for NMR instruments", J. Appl. Phys., Vol. 85, No. 8, April, 1999, p. 5205

[2] G. Friedman, "Conditions for the representation of vector hysteresis by the vector Preisach model", J. Appl. Phys., Vol. 85, No. 8, April, 1999, p. 4379

[3] G. Friedman, "A method for characterization of hysteresis in two dimensions", to appear in IEEE Transactions on Magnetics

[4] G. Friedman, " Second order Preisach model of scalar hysteresis", to appear in Physica B

[6] E.Growney, S. Grant, G. Friedman, "Calculations and experimental verification of spectral distortions due to miniature NMR probes", submitted to Journal of Magnetic Resonance

[7] S. Grant, L. Murphy, R. Magin, G. Friedman, "On the computation and experimental verification of signal to noise ratio for miniature radio frequency coils used as NMR probes", submitted to IEEE Transactions on Magnetics

[8] G. Friedman, I.D. Mayergoyz, " On the estimate of excess eddy current losses in current carrying conductors", submitted to Journal of Applied Physics

[9] Y. Huang, G.Friedman, I. Obaidat, J. Kouvel, "Identification of the switching field function of bistable elements in vector hysteresis models", submitted to Journal of Applied Physics

\section{CONFERENCE PRESENTATIONS}

a) Growney, E.; Friedman, G.; Gerald II, R.; Klingler, R.; Rathke, J.; "BOUNDARY ELEMENT TECHNIQUE FOR CALCULATIONS OF FIELD AND SPECTRAL DISTORTIONS", ISMRM Workshop on Computational Electromagnetics in Magnetic Resonance, College Station, TX. May 30 June 1, 1998.

b) Growney, E.; Friedman, G.; Gerald II, R.E.; Klingler, R.J.; Rathke, J.W.; Nunez, L.H.; Aumeier, S.E.; "NMR FOR NONDESTRUCTIVE EVALUATION OF NUCLEAR MATERIALS", NMR Symposium of the 40th Rocky Mountain Conference on Analytic Chemistry, Denver, CO. July 25-30, 1998.

c) Growney, Eric; Friedman, Gennady; Gerald II, Rex; Klingler, Robert; Rathke, Jerome; Nunez, Luis; "EVALUATION AND DESIGN OF TOROID CAVITY PROBES BASED ON CALCULATED DISTORTIONS", The 40th Experimental Nuclear Magnetic Resonance Conference, Orlando, FL. February 28 - March 5, 1999. 
d) Growney, Eric; Friedman, Gary; "COMPUTATIONS OF DISTORTIONS IN MAGNETIC FIELD SPECTRUM FOR NUCLEAR MAGNETIC RESONANCE INSTRUMENTS", The 43rd Annual Conference on Magnetism and Magnetic Materials, Miami, FL. November 9-12,1998.

e) Grant, Samuel; Growney, Eric; Magin, Richard; Friedman, G.; "SIGNAL-TO-NOISE IMPROVEMENT WITH MULTILAYERED RF MICROCOILS", The $40^{\text {th }}$ Experimental Nuclear Magnetic Resonance Conference, Orlando, FL. February, 28 - March 5, 1999. 